\title{
Estudio de la Microestructura y de las propiedades reológicas de geles de concentrado proteico de sabalo (prochilodus platensis ) en relación a la etapa de lavado con soluciones de $\mathrm{H}_{3} \mathrm{PO}_{4}$ y $\mathrm{NaHCO}_{3}$.
}

\author{
María A. Reinheimer ${ }^{1}$, José R. Medina ${ }^{* 2}$, Luciana Beresvil ${ }^{(3)}$ y Gustavo A. Pérez ${ }^{4}$ \\ (1) INGAR -CONICET-UTN, Avellaneda 3657 S3002 GJC Santa Fe-Argentina. \\ $(2,3,4)$ Facultad de Ingeniería Química - UNL - Santiago del Estero 2829 S3000 AOM Santa Fe- Argentina. \\ E-mail: jrmedina@fiq.unl.edu.ar
}

(Recibido/received: 21-Diciembre-2012; aceptado/accepted: 24-Junio-2013)

\begin{abstract}
RESUMEN
El concentrado de proteínas de pescado (CPP) es el músculo del pescado desmenuzado y exento de huesos, piel, carne oscura y de espinas, que es lavado varias veces con agua y escurrido hasta la proporción de agua original. Aquí se estudió el efecto en la composición físico-química, sobre la microestructura y las propiedades reológicas de geles de CPP utilizando diferentes soluciones de agua de lavado en la obtención de CPP de sábalo (Prochilodus platensis). Al mismo tiempo se evaluó la extracción de proteínas durante el lavado, y el análisis de las propiedades reológicas de los geles obtenidos. Los geles de CPP de sábalo obtenidos mediante el método de lavado $(0.05 \%$ H3PO4, $0.2 \%$ NaHCO3 y $0.2 \% \mathrm{NaCl}$ ) presentaron un contenido aceptable de proteínas, el menor contenido de materia grasa, y la textura más aceptable, confirmada por la reología y la microestructura, mostrando mejoras respecto de los obtenidos por el método convencional.
\end{abstract}

Palabras Clave: CPP; sábalo; microestructura; geles.

\begin{abstract}
The fish protein concentrate (FPC), is minced fish muscle and free of bones, skin, bones and dark meat, which is washed several times with water and drained to the original water ratio. The paper studies the effect on the physical and chemical composition, the microstructure and functional properties of gels FPC using different wash water solutions in obtaining tarpon FPC (Prochilodus platensis). Were evaluated protein extractions during washing, and analysis of the functional properties of the gels obtained. FPC gels obtained by the method shad wash $(0.05 \%$ $\mathrm{H} 3 \mathrm{PO} 4,0.2 \% \mathrm{NaHCO} 3$ and $0.2 \% \mathrm{NaCl}$ ) had an acceptable content of protein, lower fat content, and more acceptable texture, rheology and confirmed by microstructure, showing improvements over the gels obtained by the conventional method.
\end{abstract}

Keywords: FPC; gels; sábalo; microstructure.

\footnotetext{
* Autor para la correspondencia.
} 


\section{INTRODUCCIÓN}

El concentrado proteico de pescado (CPP) no es un alimento en sí mismo, sino que es el intermediario para formulaciones de sustitutos de mariscos. Se podría definir como un concentrado de proteínas miofibrilares del músculo de pescado, obtenido mediante el lavado del pescado refinado (sin huesos, espinas, piel y entrañas) y desmenuzado, el cual es mezclado con crioprotectores para un almacenamiento aceptable bajo congelación. Esta base o intermediario para la preparación de los productos análogos, de gran interés en el mundo occidental, podría presentarse como alternativa industrial en Argentina. Sobre todo para las especies ictícolas de agua dulce, de gran producción de biomasa, con el fin de proporcionar una forma distinta de presentación que permita una mejor conservación y por lo tanto extender su vida útil para su comercialización.

Los principales motivos del auge del CPP a nivel mundial son: el mejor aprovechamiento del recurso ictícola desde el punto de vista nutricional, la versatilidad tecnológica ya que involucra una tecnología simple y de relativamente bajos costos de inversión, ofreciendo a la vez la posibilidad de estabilizar la textura de la pulpa mediante el agregado de crioprotectores, y por último la expansión de mercados existentes y la alternativa de desarrollar nuevos productos atractivos que permitirá la ampliación del mercado de productos pesqueros (Manca y Trinchero, 1984a).

Medina (2000), Medina y Garrote (2002) y Medina y otros (2008) en su línea de investigación han planteado con sostenido interés la posibilidad de utilizar como materia prima en la obtención de CPP, especies provenientes de agua dulce, las cuales presentan disponibilidad de captura, como es el surubí, y actualmente el sábalo.

El sábalo (Prochilodus platensis) es el recurso pesquero más abundante del litoral fluvial argentino. Es un eslabón crucial en los ecosistemas que integra, dada su condición de especie forrajera, sostén de la cadena trófica. Se trata de un pez iliófago.

Es una especie naturalmente muy fecunda, y la misma forma parte de una estrategia reproductiva exitosa, adaptada a las características del régimen natural de pulsos de inundación del sistema, que involucra la realización de migraciones río arriba y el desove en aguas abiertas, acoplado a las crecientes, como mecanismo de dispersión de huevos y larvas a las _áreas de cría del valle aluvial (Espinach Ros y Sánchez, 2007). Este tipo de tecnología de producción presenta ventajas convenientes para la comercialización y explotación de productos a base de proteínas provenientes del sábalo, recurso explotado generalmente bajo su única condición de consumo en fresco.

Aunque la tecnología del CPP ha sido desarrollada y ampliamente investigada a partir de especies de mar sin valor comercial o sin explotación, actualmente se registran muy pocos trabajos que hayan utilizado como materia prima especies de pescado de agua dulce, y menos por supuesto, de alto contenido en grasa como es el sábalo, pero de una gran producción de biomasa. Todos los avances realizados se han referido en general a pescado de mar y de bajo contenido en grasa. De todos modos, investigadores japoneses han obtenido notables mejoras en la tecnología del procesamiento de especies de alto contenido en grasa, logrando CPP con propiedades funcionales excelentes, lo que abre buenas expectativas para el aprovechamiento de tales especies (Nishioka yotros, 1990).

En el presente trabajo se estudió el efecto en la composición físico-química, en la microestructura y en las propiedades funcionales de geles de CPP utilizando diferentes soluciones de agua de lavado en la obtención de CPP de sábalo. Los efectos de soluciones de $\mathrm{NaHCO}_{3}$ y $\mathrm{H}_{3} \mathrm{PO}_{4}$ fueron evaluados para ver los niveles de extracción de proteínas durante el lavado, como así también analizar las propiedades reolóligas y de la microestructura de los geles obtenidos.

\section{MATERIALES Y MÉTODOS}

\section{Materiales}

Los sábalos frescos adquiridos en el mercado local fueron procesados dentro de las $24 \mathrm{~h}$ de captura. Los pescados se conservaron cubriéndolos con hielo molido, de forma de evitar aumentos de temperatura durante el transporte, y posteriormente fueron llevados directamente al laboratorio para su procesamiento. El músculo blanco fue separado de las espinas, cuero, huesos y partes de músculo oscuro manualmente con cuchillo. El desmenuzado de sábalo fue preparado con una picadora eléctrica de carne con cuchilla y placa perforada para obtener un diámetro de partículas de 5 $\mathrm{mm}$.

\section{Preparación de la mezcla de CPP congelado}

El sistema de lavado descripto en el trabajo de Medina (2000), consistió de una cestilla cilíndrica de malla de acero inoxidable de 30 mesh, en la cual para cada experiencia se le agregaron 300 gr de desmenuzado de sábalo acondicionado a la temperatura del lavado. La cestilla con el desmenuzado fue colocada en un tubo de 
acero inoxidable. A su vez, este tubo de acero inoxidable fue colocado en un baño termostático con agua a la temperatura requerida. Inmediatamente después del lavado y el prensado, el desmenuzado fue pesado y se le incorporó los crioprotectores sacarosa y sorbitol, en una relación 1:1 (p/p), de manera tal que alcance una concentración final del $8 \%$ en el peso final del bloque y $0.2 \%$ de tripolifosfato de sodio. Después que la masa fue mezclada manualmente con los crioprotectores se formaron bloques pequeños $(3 \mathrm{~cm}$. de diámetro y $12 \mathrm{~cm}$. de largo aproximadamente), se congelaron en Nitrogeno líquido y luego se almacenaron a $-21{ }^{\circ} \mathrm{C}$ en freezer hasta la determinación de los parámetros planteados en este trabajo, de acuerdo a la metodología recomendada por Lee (1986).

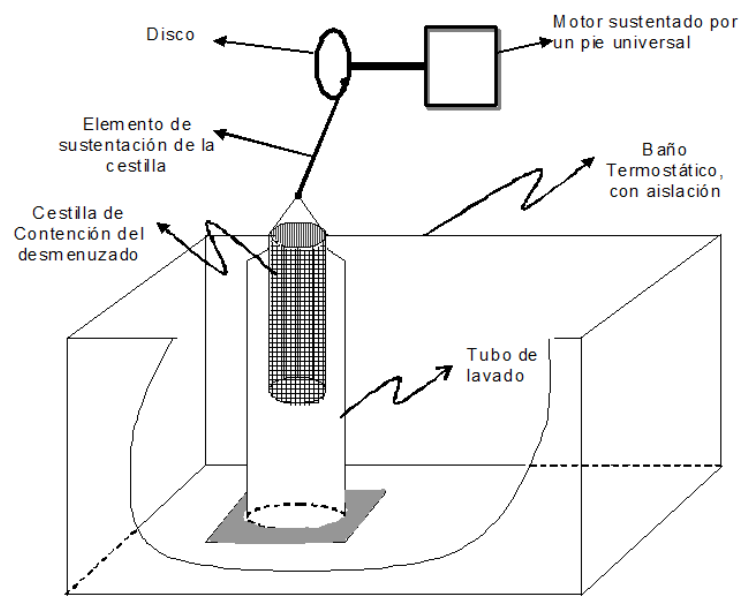

Figura 1. Esquema del equipo de lavado.

Condiciones de lavado

Las condiciones experimentales utilizadas se basaron en las obtenidas en el trabajo de Medina y Garrote (2002), en el cual se elaboró CPP a partir de surubí pintado (Pseudoplatystoma coruscans). En el mismo se pudieron predecir las mejores condiciones del lavado a escala de laboratorio asociado a un CPP de buena calidad, por medio de un modelo matemático estadístico basado en la metodología de superficie de respuesta (RSM). Los aspectos básicos y de aplicación de RSM para el diseño experimental han sido ampliamente explicados por Montgomery (1991) y Khuri y Cornell (1996).

El punto elegido del modelo que verifica los modelos de regresión analizados y con el cual se obtuvieron resultados adecuados para una buena calidad de CPP fue, $18{ }^{\circ} \mathrm{C}$ (del agua de lavado); tiempo de cada ciclo: $\mathrm{t}$ $=4.62$ minutos (con 3 ciclos de lavados) y $\mathrm{R}=3.5: 1$ (relación de agua de lavado/músculo desmenuzado).
Para las experiencias realizadas a partir de sábalo, se partió de las condiciones anteriores, y se analizaron variaciones en la temperatura y tiempo de lavado alrededor del punto de regresión obtenido sobre la calidad del producto. Durante el análisis pudo verificarse y compararse que bajo las mismas condiciones de lavado, los músculos de ambas especies se comportaban de manera similar (Medina y otros, 2010); y a su vez se probó que bajo las condiciones de operación de la etapa de lavado de: Temperatura agua de lavado $=18{ }^{\circ} \mathrm{C} ; \mathrm{t}=4.62$ minutos/ciclo (con 3 ciclos de lavados) y $\mathrm{R}=3.5: 1$ (relación de agua de lavado/músculo desmenuzado), se obtuvieron geles de CPP de sábalo de aceptable calidad (Medina y otros (2008); Medina y otros (2010)). A partir de estas condiciones de operación, se estudiaron los factores que modificaban los componentes del músculo original en el proceso de obtención del CPP al variar la composición del agua de lavado en los diferentes ciclos.

La Tabla 1 presenta las diferentes experiencias llevadas a cabo, siempre desarrolladas bajo las mismas condiciones de operación detalladas, que serán comparadas con el CPP obtenido por el proceso convencional de lavado (muestra de control, C)

Tabla 1. Tratamientos experimentados en la etapa de lavado

\begin{tabular}{|c|c|l|l|l|l|}
\hline & \multicolumn{5}{|c|}{ Experimento } \\
\hline & C & \multicolumn{1}{|c|}{ E1 } & \multicolumn{1}{|c|}{ E2 } & \multicolumn{1}{c|}{ E3 } & \multicolumn{1}{|c|}{ E4 } \\
\hline $\mathbf{1}^{\mathbf{0}}$ & A.D. & $0.5 \%$ & $0.05 \%$ & $0.05 \%$ & $0.05 \%$ \\
Ciclo & & $(\mathrm{p} / \mathrm{p})$ & $(\mathrm{v} / \mathrm{v})$ & $(\mathrm{v} / \mathrm{v})$ & $(\mathrm{v} / \mathrm{v})$ \\
& & $\mathrm{NaHCO}_{3}$ & $\mathrm{H}_{3} \mathrm{PO}_{4}$ & $\mathrm{H}_{3} \mathrm{PO}_{4}$ & $\mathrm{H}_{3} \mathrm{PO}_{4}$ \\
\hline $\mathbf{2}^{\mathbf{o}}$ & A.D. & $0.5 \%$ & $0.05 \%$ & $0.5 \%$ & $0.2 \%$ \\
Ciclo & & $(\mathrm{p} / \mathrm{p})$ & $(\mathrm{v} / \mathrm{v})$ & $(\mathrm{p} / \mathrm{p})$ & $(\mathrm{p} / \mathrm{p})$ \\
& & $\mathrm{NaHCO}_{3}$ & $\mathrm{H}_{3} \mathrm{PO}_{4}$ & $\mathrm{NaHCO}$ & $\mathrm{NaHCO}$ \\
\hline $\mathbf{3}^{\mathbf{o}}$ & $0.2 \%$ & $0.2 \%$ & $0.2 \%$ & $0.2 \%$ & $0.2 \%$ \\
Ciclo & $(\mathrm{p} / \mathrm{p})$ & $(\mathrm{p} / \mathrm{p})$ & $(\mathrm{p} / \mathrm{p})$ & $(\mathrm{p} / \mathrm{p})$ & $(\mathrm{p} / \mathrm{p})$ \\
& $\mathrm{NaCl}$ & $\mathrm{NaCl}$ & $\mathrm{NaCl}$ & $\mathrm{NaCl}$ & $\mathrm{NaCl}$ \\
\hline
\end{tabular}

\section{Preparación de Geles}

Las muestras de CPP congeladas fueron atemperadas a $20 \pm 2{ }^{\circ} \mathrm{C}$ por $2 \mathrm{hrs}$ hasta alcanzar una temperatura en la capa superficial de $-3{ }^{\circ} \mathrm{C}$. Los bloques fueron luego cortados en rodajas de espesores regulares y se picaron a presión atmosférica con una picadora utilizando una placa perforada de $7 \mathrm{~mm}$ de diámetro. De esta manera, se logró reducir el tamaño y mejorar el proceso de homogeneizado con los ingredientes.

Posteriormente, se procedió al mezclado mediante la incorporación de $2.5 \%$ de $\mathrm{NaCl}$ sobre la base del peso 
final del sol (mezcla del músculo lavado más el agregado de sales y crioprotectores) y de la cantidad de hielo necesaria para ajustar la humedad final a $74 \%$, durante 9-10 min para lograr la solubilización de las proteínas. Esta mezcla fue luego embutida en moldes cilíndricos de acero inoxidable de $3 \mathrm{~cm}$ de largo por 3 $\mathrm{cm}$ de diámetro. El último paso fue el tratamiento térmico para lograr la gelificación, que consistió de dos etapas: un primer tratamiento de asentamiento (setting) o proceso térmico diferencial de 21 minutos a $40{ }^{\circ} \mathrm{C}$ en un baño termostático con agua continuamente agitado, e inmediatamente se procedió a la cocción final de 21 minutos a $90{ }^{\circ} \mathrm{C}$ en otro baño termostático con agitación. Por último, los tubos fueron enfriados en una batea con agua y hielo por $20 \mathrm{~min}$, se extrajeron los geles cilíndricos de los moldes por medio de un pistón extractor y fueron envueltos en papel de aluminio, rotulado y almacenado en freezer hasta su evaluación reológica.

\section{Composición Química}

Para caracterizar químicamente el material crudo fresco y el CPP se determinó proteína cruda total ( $\mathrm{N}$ x 6.25) por método de Kjeldhal (AOAC, 1995), grasa total (Folch y otros, 1957), cenizas totales (AOAC, 1995) y humedad (Lanier y otros, 1995)

\section{Eficiencia de lavado}

La eficiencia de la etapa de lavado fue estimada como el porcentaje de recuperación de proteínas totales (Pacheco-Aguilar y otros, 2001):

$\%$ Proteínas totales recuperadas $=($ cantidad de proteínas recuperadas luego del lavado) / (cantidad de proteínas del músculo picado sin lavar) $\%$

\section{Mediciones de resistencia de gel}

La resistencia del gel (gel strength, GS) se obtuvo como el producto del esfuerzo de corte (primer pico en la gráfica y la deformación, distancia al primer pico de la gráfica). Las determinaciones se realizaron por cuadriplicado. Las probetas de gel fueron piezas cilíndricas de $3 \mathrm{~cm}$ de diámetro por $3 \mathrm{~cm}$ de alto, atemperadas a $20{ }^{\circ} \mathrm{C}$ previo a la medición de la resistencia. El ensayo de penetración o punción (para determinar la resistencia a la rotura del gel) fue medida usando una máquina universal Instron modelo 3344 con sistema de simple columna (Instron Ltd., USA) equipada con una punta de cabezal cilíndrico (diámetro: $7 \mathrm{~mm}$ ). El ensayo fue realizado usando una velocidad del cabezal de $60 \mathrm{~mm} \mathrm{~min}^{-1}$. El punto de parada en el descenso del cabezal de la máquina universal fue fijado de tal modo que la punta de la probeta quedara a $1 \mathrm{~cm}$ de la plataforma metálica que sostenía la muestra. La muestra o espécimen de cada CPP fue colocado directamente debajo de la probeta de manera que la punción se efectuara en el centro axial de la muestra (Lanier y otros, 1985).

\section{Microscopía Electrónica de Barrido (SEM)}

La microestructura de los geles de CPP fue observada mediante SEM. Láminas de gel de $2-3 \mathrm{~mm}$ de espesor fueron fijadas con glutaraldehído al $2.5 \%$ (v/v) en buffer fosfato $0.2 \mathrm{M}(\mathrm{pH} 7.2)$ durante 12 horas. Las muestras fueron luego enjuagadas dos veces, primero en buffer por $30 \mathrm{~min}$ y luego en agua destilada por $30 \mathrm{~min}$ antes de ser deshidratadas en etanol con concentraciones crecientes de: $50 \%, 70 \%, 80 \%, 90 \%, 100 \%, 100 \%$ y $100 \%(\mathrm{v} / \mathrm{v})$ durante $30 \mathrm{~min}$ en cada concentración. Las muestras deshidratadas fueron montadas sobre tacos para SEM utilizando pintura conductora de plata, luego secadas y recubiertas con oro utilizando un evaporador de laboratorio VEECO modelo VE-300 (Veeco Instruments Inc., Long Island, NY, USA). Las observaciones se realizaron utilizando un microscopio electrónico de barrido JEOL JSM-35C (JEOL Ltd., Tokyo, Japan) operado bajo el modo de imágenes de electrones secundarios utilizando una tensión de aceleración de $20 \mathrm{kV}$, a distintas magnificaciones (X): 100,480 y 1000.

\section{Análisis estadístico}

Los datos experimentales fueron calculados como valores medios \pm desviación estándar. Las diferencias entre las medias fueron comparadas por el método de diferencia mínima significativa $(\mathrm{p}<0.05)$ y calculadas usando Statgraphics 5.1 Software (Manugistics, Rockville, MD, USA).

\section{RESULTADOS Y DISCUSIÓN}

\section{Composición química y rendimientos del lavado}

En la Tabla 2, se puede observar la diferencia de los tratamientos realizados sobre los macronutrientes del CPP obtenido. Los resultados reportados en la Tabla 2 no muestran el contenido de carbohidratos, que es igual al proveniente del agregado de los crioprotectores en todas las muestras de CPP (8\% de sacarosa-sorbitol). 
Tabla 2 - Composición aproximada de sábalo fresco molido y los diferentes CPP

\begin{tabular}{ccccc}
\hline & \multicolumn{4}{c}{ Composiciones (\% base húmeda) $^{\mathbf{A}}$} \\
\cline { 2 - 5 } & Humedad & $\begin{array}{c}\text { Proteína } \\
\text { cruda }\end{array}$ & $\begin{array}{c}\text { Grasa } \\
\text { Cruda }\end{array}$ & Cenizas \\
\hline Músculo fresco & $73.46 \pm 0.45^{\mathrm{a}}$ & $17.11 \pm 0.22^{\mathrm{a}}$ & $9.42 \pm 0.11^{\mathrm{b}}$ & $0.88 \pm 0.007^{\mathrm{a}}$ \\
C & $74.74 \pm 0.07^{\mathrm{b}}$ & $11.84 \pm 0.01^{\mathrm{b}}$ & $4.37 \pm 0.11^{\mathrm{a}}$ & $0.30 \pm 0.01^{\mathrm{b}}$ \\
E1 & $80.61 \pm 0.29^{\mathrm{b}}$ & $6.30 \pm 0.09^{\mathrm{c}}$ & $8.65 \pm 0.12^{\mathrm{b}}$ & $0.97 \pm 0.01^{\mathrm{a}}$ \\
E2 & $73.33 \pm 0.41^{\mathrm{a}}$ & $12.62 \pm 0.17^{\mathrm{b}}$ & $3.25 \pm 0.35^{\mathrm{a}}$ & $2.82 \pm 0.06^{\mathrm{a}}$ \\
E3 & $72.26 \pm 0.49^{\mathrm{a}}$ & $11.68 \pm 0.24^{\mathrm{b}}$ & $4.86 \pm 0.26^{\mathrm{a}}$ & $2.93 \pm 0.08^{\mathrm{a}}$ \\
E4 & $75.57 \pm 0.32^{\mathrm{a}}$ & $12.49 \pm 0.29^{\mathrm{b}}$ & $1.69 \pm 0.52^{\mathrm{a}}$ & $2.88 \pm 0.05^{\mathrm{a}}$ \\
\hline
\end{tabular}

El mismo superíndice en la misma columna indica que no hay diferencias significativas ( $p>0.05)$.

${ }^{\mathrm{A}}$ Medias \pm DS de muestras por triplicado.

Como puede desprenderse de la Tabla 2, el sábalo es una especie de alto contenido de materia grasa. El particular inconveniente relacionado con alto contenido de grasa, son las reacciones de oxidación de lípidos que pueden manifestarse, y como consecuencia, la carne de pescado desprende mal olor y se decolora (Sonu (1986); Lanier y otros (2000)). Esto enfatiza lo crucial que resulta la etapa de lavado para la reducción de su contenido como así también de componentes indeseables, como son las proteínas solubles. Por lo tanto, se procedió a variar la formulación de las soluciones de lavado y de esta manera, analizar el impacto que tienen en la calidad de los geles.

La Figura 2 presenta el efecto de los tratamientos de lavados sobre el \% de recuperación de proteínas.

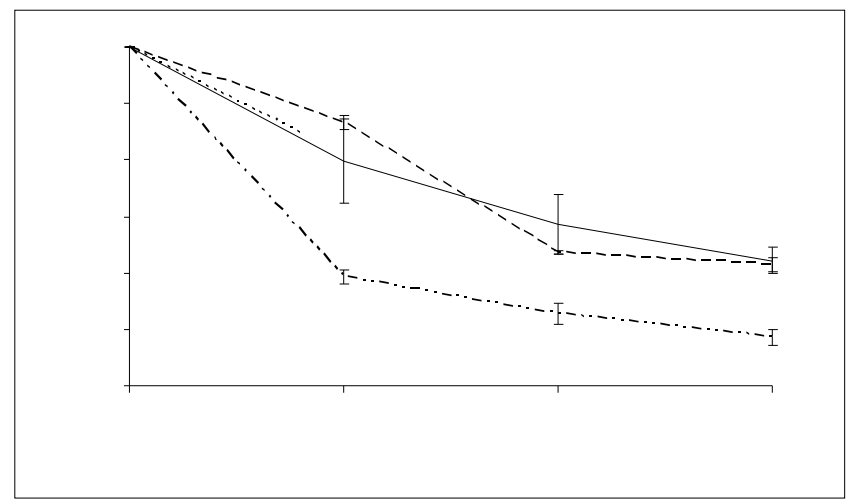

Figura 2 - Efecto de las diferentes soluciones de lavado sobre el porcentaje de recuperación de proteínas del músculo molido previo al lavado

Luego del tratamiento los geles de la experiencia 1 (E1) no alcanzaron a desarrollarse. Este fenómeno pudo deberse a que el contenido de proteínas extraídas en el lavado fue excesivo, de esta forma, el contenido mínimo requerido para formar el entrecruzamiento en el gel no fue alcanzado. Por lo tanto, la combinación de soluciones de lavado utilizadas en E1, extrajeron no solo las proteínas sarcoplasmáticas sino que también parte de las miofibrilares, que son las responsables de la capacidad de gelficación del CPP. Por consiguiente, como muestra la Figura 2, las mayores pérdidas ocurrieron en la experiencia E1. Con este resultado, se concluye que el método de lavado utilizado en E1, usando 2 ciclos de lavado de $0.5 \%$ (p/p) NaHCO3, no es apropiado para elaborar CPP utilizando sábalo. Sin embargo Suzuki (1981) hace una sugerencia de realizar lavados con bicarbonato de sodio sin especificar las condiciones para peces grasos. Además, como se puede apreciar en la Tabla 2, para E1 se obtuvo el contenido más alto de materia grasa. Desde el punto de vista de la formación del gel, la materia grasa interfiere en la formación de la matriz de proteínas, dificultando la gelificación. Los resultados obtenidos en E1 difieren respecto a los obtenidos en los trabajos de Nakamura y otros (1978) e Ishikawa y otros (1979), donde resultados satisfactorios fueron obtenidos en la remoción de grasa y obtención de geles con mejores propiedades para especies de pescado con alto contenido de grasa, similar al sábalo y utilizando las mismas composiciones de soluciones de lavado. Aunque los resultados obtenidos de E1 y también la baja resistencia de gel obtenida en E3 concuerdan con las obtenidas para pescado y otras especies de carne en los trabajos de Niwa y Mussato (1971), Babji et al. (1995) y Ng y Huda (2011). Dado que $0.5 \% \mathrm{NaHCO}_{3}$ es un agente reductor que interfiere con los enlaces - $\mathrm{SH}$ de las proteínas del músculo haciendo más débil la resistencia de los geles de proteínas (Niwa \& Musato, 1971).

Los geles obtenidos en los tratamientos de lavado del desmenuzado de sábalo de E2 a E4 presentan adecuados resultados comparados con la muestra de control. La humedad final obtenida es cercana al valor de humedad 
original del músculo fresco, lo cual concuerda con la conclusión de Sierra y otros (1991) donde el valor óptimo de $\mathrm{NaCl}$ utilizado es del $0.2 \%$ en la solución de lavado del 3o ciclo, de manera tal de obtener un CPP con una humedad cercana al original de músculo fresco y cuyos parámetros de calidad no disminuyeron, evitando la extracción de proteínas miofibrilares, concordando también con la propuesta de Lee (1986) que recomienda un rango de $0.1-0.2 \%$ de $\mathrm{NaCl}$. Los contenidos de proteínas en todos los geles fueron similares. Esto también se ve reflejado en los valores similares de eficiencia en la recuperación de proteínas de la etapa de lavado (Figura 2). Sin embargo, se obtuvieron diferencias en el contenido de grasa. Los geles desarrollados a partir de las condiciones planteadas en E4, presentaron uno de los valores más altos de proteínas y el menor contenido de materia grasa.

Efecto de las diferentes soluciones de lavado en los valores de resistencia de gel y deformación.

En esta sección se procederá a comparar los valores de esfuerzo de corte y deformación de los geles. Dichos valores son presentados en la Figura 3.

Los geles de CPP obtenidos por el método convencional, C, mostraron aceptable capacidad de formación de gel. Los resultados de resistencia de gel mostrados aquí concuerdan con los reportados en otros trabajos donde se han utilizados especies de pescado de río y con presencia de músculo oscuro (Sonu, 1986; Luo et al., 2008; Rawdkuen et al., 2009).

Similares valores de esfuerzo de corte y deformación $(p<0.05)$ fueron hallados para las muestras E4. Sin embargo, los menores valores fueron obtenidos para la experiencia E3, que solo difiere de la E4 en la concentración de la solución utilizada en el segundo ciclo. Estos resultados y la observación de la microestructura, sugieren que los menores valores de la resistencia de corte pueden deberse a que las estructuras fibrilares aparecen en forma individual (o zonas de agregaciones desordenadas). Además, está demostrado que el aumento en la concentración de proteínas, incrementa la resistencia del gel, de acuerdo a las conclusiones reportadas por Luo y otros (2008).

Es importante destacar que el contenido de materia grasa es un factor crítico que puede afectar la resistencia del gel (Park y otros, 2005). Por lo tanto, el bajo contenido de grasa en los geles obtenidos en $\mathrm{E} 4$, puede contribuir a que presenten un mayor valor de resistencia, y con el mismo análisis, el alto contenido de grasa presente en los geles E3, puede ser responsable de que presenten menor valor de esfuerzo de corte.
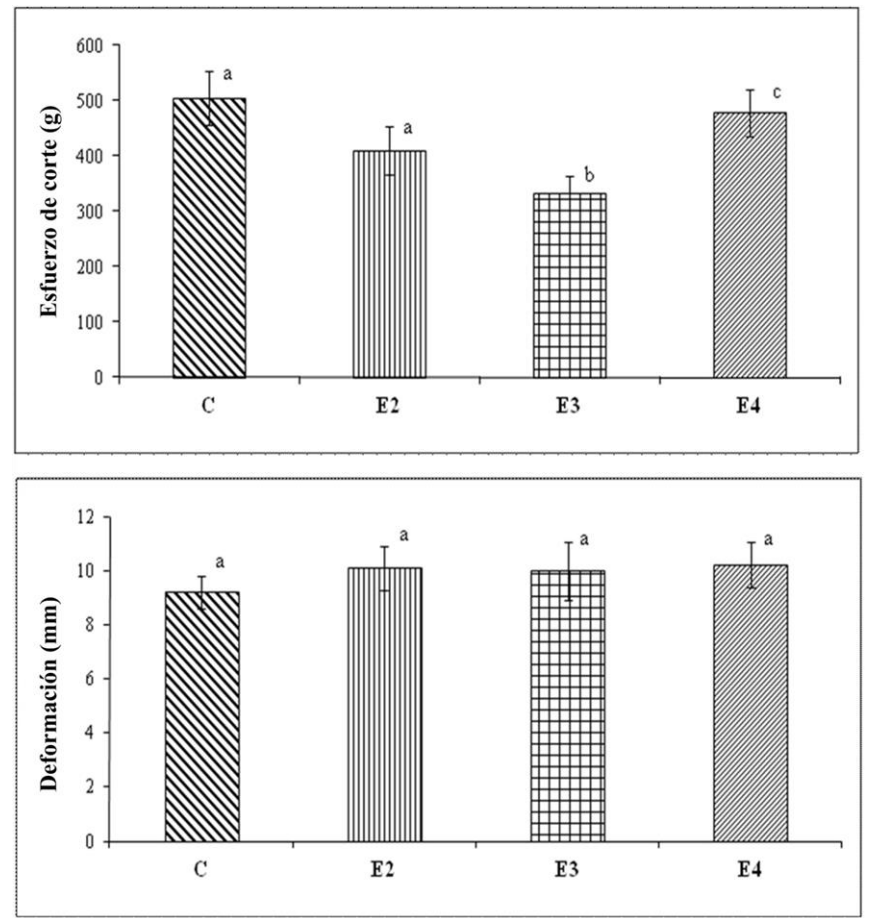

Figure 3 Esfuerzo de corte y deformación.

\section{Microestructura}

La microestructura de los geles de CPP obtenidos en las experiencias E2 a E4 fue observada mediante SEM, como se observan en las Figuras 4 y 5 . A baja magnificación (100x) (resultados no mostrados), existió poca diferencia entre las muestras.

La microestructura de las muestras exhibieron una matriz del gel compacta y porosa con numerosos huecos. Sin embargo, la red del gel en E4 resultó ser más compacta y densa y a su vez con estructura homogénea en todos los poros.

A mayores magnificaciones (480x y 1000x), E2 y E4 presentaron estructuras fibrilares que fueron más compactas que las observadas en E3, debido a una mayor asociación entre las fibras adyacentes (Figuras 4 y 5). E3 mostró una morfología en la cual zonas fibrosas coexistieron con otras zonas de agregados desordenados. En algunas zonas, fue evidente la estructura de entrecruzamiento desordenado con apariencia globular en la superficie. Como fue mencionado anteriormente, estas áreas de agregaciones desordenadas tienden a que se produzcan bajos valores de resistencia del gel (Alvarez y otros, 1999). 
La estructura de los geles obtenidos en E4 está compuesta por la asociación de fibras y poros de forma y tamaño homogéneos que concuerdan con la alta resistencia del gel. De esta forma, el arreglo y asociación de las moléculas de proteínas en la matriz del gel directamente contribuyen a la resistencia del gel del CPP de sábalo. En el trabajo de Rawdkuen y otros (2009), resultados similares fueron obtenidos acerca de la influencia de la estructura regular de la matriz del gel sobre los valores de resistencia. La estructura ordenada es atribuible de una alta resistencia de gel.
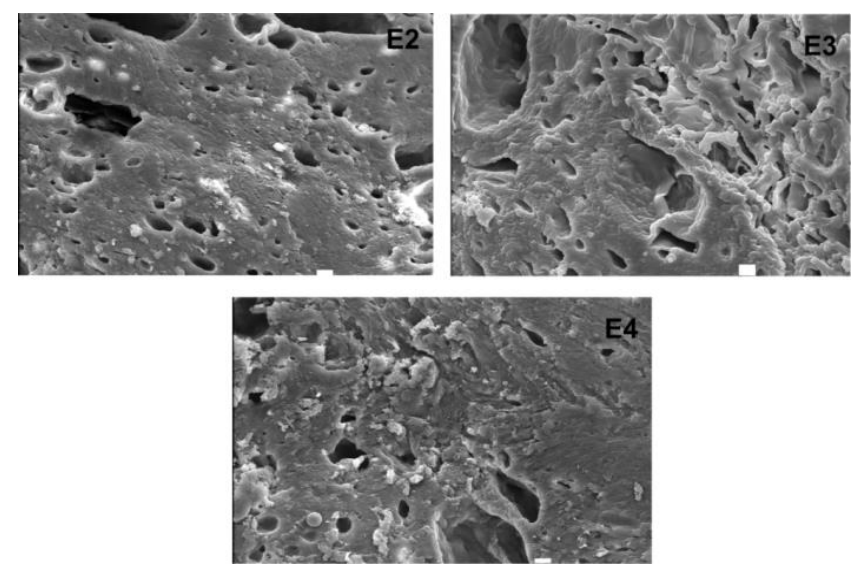

Figura 4. Imagen obtenida por Microscopía Electrónica de Barrido (Aumento: 480x) La barra blanca representa $10 \mu \mathrm{m}$.
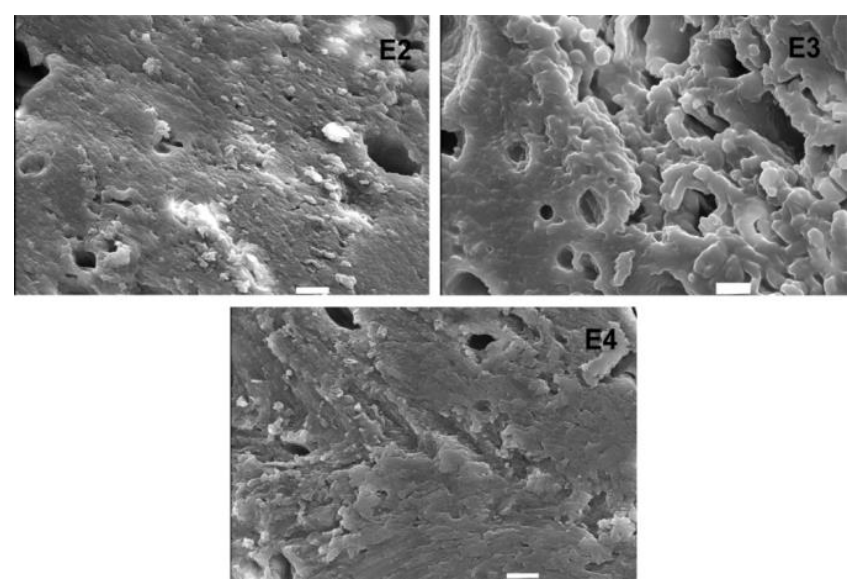

Figura 5 Imagen obtenida por Microscopía Electrónica de Barrido (Aumento: 1000x) La barra blanca representa $10 \mu \mathrm{m}$.

\section{Conclusiones}

La carne proveniente de sábalo resulta apta como materia prima para la elaboración de geles de CPP, conclusión formulada a partir de los valores de resistencia de gel obtenidos. La elección de las soluciones de lavado en la tecnología de elaboración de
CPP tiene significativa influencia sobre el contenido de proteínas y lípidos, cuando se procesa carne de pescado de especies de río como es el sábalo. Los experimentos realizados sugirieron, que el contenido de proteínas y grasa son los factores más influenciables sobre los valores de resistencia del gel.

Bajo el rango de condiciones estudiadas, los geles de CPP de sábalo obtenido mediante el método de lavado E4 $(0.05 \%$ H3PO4, $0.2 \%$ NaHCO3 y $0.2 \% \quad \mathrm{NaCl})$ presentaron un contenido aceptable de proteínas, el menor contenido de materia grasa, como así también, la textura más aceptable, tal como fue confirmado por análisis reológicos y de microestructura, mostrando mejoras respecto de los geles obtenidos por el método convencional de lavado.

Los resultados obtenidos en la experiencia E1 (método de lavado de 2 ciclos de $0.5 \%$ NaHCO3 y 1 de $0.2 \%$ $\mathrm{NaCl}$ ), sugirieron que el método no fue apropiado para músculo molido de sábalo, resultando un producto más parecido a una emulsión que a un gel, debido al alto contenido de materia grasa.

\section{CONCLUSIÓN}

La carne proveniente de sábalo resulta apta como materia prima para la elaboración de geles de CPP, conclusión formulada a partir de los valores de resistencia de gel obtenidos. La elección de las soluciones de lavado en la tecnología de elaboración de CPP tiene significativa influencia sobre el contenido de proteínas y lípidos, cuando se procesa carne de pescado de especies de río como es el sábalo. Los experimentos realizados sugirieron, que el contenido de proteínas y grasa son los factores más influenciables sobre los valores de resistencia del gel.

Bajo el rango de condiciones estudiadas, los geles de CPP de sábalo obtenido mediante el método de lavado E4 $(0.05 \%$ H3PO4, $0.2 \%$ NaHCO3 y $0.2 \% \quad \mathrm{NaCl})$ presentaron un contenido aceptable de proteínas, el menor contenido de materia grasa, como así también, la textura más aceptable, tal como fue confirmado por análisis reológicos y de microestructura, mostrando mejoras respecto de los geles obtenidos por el método convencional de lavado.

Los resultados obtenidos en la experiencia E1 (método de lavado de 2 ciclos de $0.5 \%$ NaHCO3 y 1 de $0.2 \%$ $\mathrm{NaCl}$ ), sugirieron que el método no fue apropiado para músculo molido de sábalo, resultando un producto más 
parecido a una emulsión que a un gel, debido al alto contenido de materia grasa.

\section{REFERENCIAS}

Microstructure of suwari and kamaboko sardine surimi gels, Journal of the Science of Food and Agriculture, $\mathrm{n}^{\circ}$ 79 , pp. 839-844.

AOAC, Official Methods of Analysis (16th ed. 1995), Washington, DC: Association of Official Analytical Chemists.

Babji, A. S., Mukhlis, I., Gna, S. K., Seri Chempaka, M. Y., Norhaliza, M. and Eraou, B.(1995) Processing efficiency and physicochemical properties of surimitype materials, Malaysian Journal of Animal Science, $\mathrm{n}^{\circ} 1$, pp. 52-58.

Box, G.E.P. y Behnken, DW (1960). Some new three level designs for the study of quantitative variables. Technometrics, 2(4), pp. 455-475.

Espinach Ros, A. and Sánchez, R.P. (2007) (Eds.), Proyecto Evaluación del Recurso Sábalo en el Paraná Informe final de los resultados de la primera etapa 20052006 y medidas de manejo recomendadas, Secretaría de Agricultura, Ganadería, Pesca y Alimentos, Subsecretaría de Pesca y Acuicultura.

Ishikawa, S., Nakamura, K., Fujii, Y., Yamano, G., Sugiyama, T., Shinozaki, K., Tobita, K. and Yamaguchi, Y. (1979). Fish jelly product (Kamaboko) and frozen minced meat (frozen Surimi) made of sardine, 3: Influence of the treatment methods for materials just after catch on the Kamaboko forming ability of sardine meat. Bulletin of Tokai Regional Fisheries Research Laboratory, Vol. 99, pp. 31-42.

Folch, J, Lees., M, Sloane-Stanley.(1957) “A simple method for the isolation and purification of total lipids from animal tissues". J. Biol. Chem, pp. 226-497.

Khuri, A.I. y Cornell, J.A. (1996). Response surfaces: designs and analyses. CRC.

Lanier, T. C., Hamann, D. D. and Wu, M. C. (1985). Development of methods for quality and functionality assessment of surimi, Final Report for Alaska Fisheries Development Foundation, Inc., Anchorage, Alaska.
Lanier, T. C. (2000). Surimi gelation chemistry, in Park, J. W. (Ed.), Surimi and Surimi Seafood, Marcel Dekker, New York, NY, pp. 237-265.

Lee, C. M (1986).Surimi manufacturing and fabrication of surimi based products, Food Technology, Vol. 40 No. 3, pp. 115-124.

Luo, Y., Shen, H., Pan, D. and Bu, G. (2008) Gel properties of surimi from silver carp (Hypophthalmichthys molitrix) as affected by heat treatment and soy protein isolate, Food Hydrocolloids, No. 22, pp. 1513-1519.

Manca, E.A. y Trinchero, J.L. (1984). Desmenuzado de pescado: tecnología y posibles usos (Parte I). La Industria Cárnica Latinoamericana, Vol. 56, pp. 41-50.

Medina, J.R. (2000). Estudio de la etapa de lavado en la obtención de surimi congelado a partir de pescado de río. Tesis doctoral, MS thesis. Universidad Nacional del Litoral. Santa Fe, Argentina.

Medina, J. R. and Garrote, R. L. (2002) Determining washing conditions during the preparation of frozen surimi from Surubí (Pseudoplatystome coruscans) using response surface methodology, Journal of Food Science, Vol. 67, No. 3, pp. 1455-1461.

Medina, J.R.; Freyre, M.R.; Piagentini, M.A. y Pérez, G.A. (2008). Efecto de las condiciones del lavado sobre la composición de nutrientes en surimi obtenido a partir de pescado de río. En: XVII Congreso Brasilero de Ingeniería Química (XVII COBEQ).

Medina, J. R., Reinheimer, M. A., Freyre, M. R. and Pérez, G. A. (2010) Cambios en la composición y de los ácidos grasos del surimi elaborado a partir de sábalo (Prochilodus platenses) modificando las condiciones de la etapa de lavado, Revista Mexicana de Ingeniería Química, Vol. 9, No. 2, pp. 159-166.

Montgomery, D. (1991). Diseño y análisis de experimentos. Grupo Editorial Iberoamérica SA, México.

Nakamura, K., Fujii, Y. and Ishikawa, S. (1978). Studies on salted and dried sardine, 1: Changes of the chemical components in sardine meat during salting, drying and storage, Bulletin of Tokai Regional Fisheries Research Laboratory, Vol. 95, pp. 75-84. 
Ng, X. Y. and Huda, N. (2011) Thermal gelation properties and quality characteristics of duck surimi-like material (duckrimi) as affected by the selected washing processes, International Food Research Journal, Vol. 18, pp. 731-740.

Nishioka, F. and Tokunaga, T.(1990) Development of New Leaching Technology and a System to Manufacture High Quality Surimi, in Proceedings of International Institute of Refrigeration, No. 3, pp. 123130.

Niwa, E. and Musato, M (1971) Physicochemical behavior of fish meat proteins - II. Reactivities of side groups of polypeptide chains during setting of fish meat paste, Bulletin Japan Society Science Fish, No. 37, pp. 884-885.

Pacheco-Aguilar, R., Ramírez-Suárez, J. C., and Mazorra-Manzano, M. A. (2001) Effect of alkaline and acidic wash treatments on functional properties and color of Monterey sardine (Sardinops sagax caerulea) minced flesh, Journal of Aquatic Food Product Technology, No. 10, pp. 85-99.

Park, J. W., Yongsawatdigul J. and Lin, T. M. (2005) Surimi: Manufacturing and evaluation, Park, J. W. (Ed.), Surimi and Surimi Seafood, Boca Raton: Taylor and Francis Group, pp. 33-106.

Rawdkuen, S., Sai-Ut, S., Khamsorn, S., Chaijan, M. and Benjakul, S.(2009) Biochemical and gelling properties of tilapia surimi and protein recovered using an acid-alkaline process, Food Chemistry, Vol. 122, No. 1, pp. 112-119.

Sierra, M. G., Jarufe, J. L., Ellenberg, I., Godoy, F. and Zapata, E. (1991) Optimización del proceso de obtención del surimi de recortes de filetes de merluza (Merluccius australis) congelados, Revista de Agroquímica y Tecnología de Alimentos, Vol. 31 No. 4, pp. 505-511.

Sonu, S. C. (1986) Surimi, NOAA Technical Memorandum NMFS, US Department of Commerce.

Susuki, T. (1981). Fish and krill protein: processing technology. Applied Science Publishers Ltd., England.

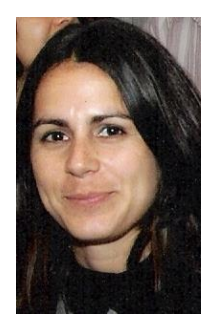

María Agustina Reinheimer es Doctora en Tecnología Química e Ingeniera en Alimentos y Becaria post-Doctoral Tipo I de CONICET (Argentina). Desarrolla de Tareas de Investigación en el CAIMI Centro de Aplicaciones Informáticas y Modelado en Ingeniería- Facultad Regional Rosario, Universidad Tecnológica Nacional, Rosario- Argentina.

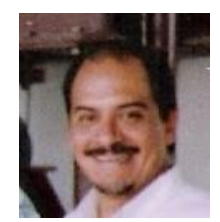

José R. Medina es Ingeniero Químico y Magister en Ciencia y Tecnología de Alimentos, recibido en la Universidad Nacional de Litoral (Argentina). Participa como Profesor de las asignaturas Ingeniería Económica y Proyecto Industrial, y con tareas de investigación sobre Tecnologías de procesamiento y conservación de matrices cárnicas.

Luciana Beresvil es graduada reciente en Ingeniería en Alimentos y realizó una pasantía de investigación en el Laboratorio de Estudios Fisicoquímicos de Alimentos perteneciente al Instituto de Tecnología de Alimentos Facultad de Ingeniería Química de la Universidad Nacional del Litoral (Argentina).

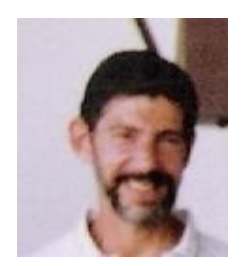

Gustavo A. Pérez es Ingeniero Químico, recibido en la Universidad Nacional del Litoral (Argentina). Desarrolla sus actividades como Profesor Titular Responsable de las asignaturas Ingeniería Económica y Proyecto Industrial en la Facultad de Ingeniería Química. Además realiza tareas de investigación en el área de "Ingeniería de Procesos". 